\title{
Criminologie
}

\section{Jeunes à risque ? Sens des pratiques dites à risque et sortie de} la rue

\section{Annamaria Colombo}

Volume 43, numéro 1, printemps-été 2010

Les jeunes et la rue

URI : https://id.erudit.org/iderudit/044055ar

DOI : https://doi.org/10.7202/044055ar

Aller au sommaire du numéro

\section{Éditeur(s)}

Les Presses de l'Université de Montréal

ISSN

0316-0041 (imprimé)

1492-1367 (numérique)

Découvrir la revue

Citer cet article

Colombo, A. (2010). Jeunes à risque ? Sens des pratiques dites à risque et sortie de la rue. Criminologie, 43(1), 155-170. https://doi.org/10.7202/044055ar

\section{Résumé de l'article}

Face à l'abondance de chiffres concernant les risques que les jeunes encourent dans la rue ou font encourir aux autres, on peut avoir tendance à oublier que leurs comportements dits " à risque » constituent les manifestations d'enjeux plus profonds. Alors que l'idéal d'autoréalisation individuelle valorise certaines formes de prise de risques, on constate simultanément la présence importante au sein de nos sociétés d'une obsession sécuritaire qui vise la gestion des populations à risque. À partir d'une enquête auprès de jeunes sortis de la rue réalisée à Montréal, le présent article vise à mettre en lumière l'écart entre le sens attribué par les jeunes à la marginalité et à la normalité et la tendance politique à privilégier des approches épidémiologique et sécuritaire à l'égard de ces populations. Il se conclut par une analyse des effets de ces approches en termes de reconnaissance sur le processus de sortie de la rue de ces jeunes. 


\title{
Jeunes à risque? Sens des pratiques dites à risque et sortie de la rue
}

\author{
Annamaria Colombo \\ Professeure \\ Responsable du Réseau d'études des confins de la santé et du social (RECSS) \\ Haute école fribourgeoise de travail social (Suisse) \\ annamaria.colombo@hef-ts.ch
}

\begin{abstract}
RÉSUMÉ - Face à l'abondance de chiffres concernant les risques que les jeunes encourent dans la rue ou font encourir aux autres, on peut avoir tendance à oublier que leurs comportements dits «à risque» constituent les manifestations d'enjeux plus profonds. Alors que l'idéal d'autoréalisation individuelle valorise certaines formes de prise de risques, on constate simultanément la présence importante au sein de nos sociétés d'une obsession sécuritaire qui vise la gestion des populations à risque. À partir d'une enquête auprès de jeunes sortis de la rue réalisée à Montréal, le présent article vise à mettre en lumière l'écart entre le sens attribué par les jeunes à la marginalité et à la normalité et la tendance politique à privilégier des approches épidémiologique et sécuritaire à l'égard de ces populations. Il se conclut par une analyse des effets de ces approches en termes de reconnaissance sur le processus de sortie de la rue de ces jeunes.
\end{abstract}

MOTS-CLÉs • Jeunes à risque, jeunes de la rue, épidémiologie, sécurité, marginalité.

\section{Introduction $^{1}$}

Les constatations des analyses indiquent que les jeunes de la rue présentent des taux élevés d'ITS [infections transmissibles sexuellement] et d'infections transmissibles par le sang. L'étude de sous-groupes dans l'échantillon des jeunes de la rue, comme les utilisateurs de drogues par injection, révèle une prévalence particulièrement inquiétante de certains agents infectieux, par exemple le virus de l'hépatite C. Elle fait également ressortir, dans cette

1. Je tiens à remercier le Conseil de recherches en sciences humaines du Canada (CRSH) pour son soutien financier à la recherche dont sont issus les résultats présentés dans cet article, ainsi que les évaluateurs anonymes de cet article pour leurs commentaires détaillés et enrichissants. 
population, des comportements sexuels à risque élevé, comme l'utilisation peu fréquente du condom et un nombre élevé de partenaires sexuels ainsi que des taux de toxicomanie préoccupants. (Agence de santé publique du Canada, 2006b: ix)

Cet extrait d'un rapport de l'Agence canadienne de santé publique sur les jeunes de la rue est révélateur d'une tendance actuelle concernant les représentations relatives à cette population dans les pays du Nord. Définis comme une population particulièrement «vulnérable» et même «à risque élevé», les jeunes de la rue et leur réalité sont en effet majoritairement abordés dans les écrits dans une perspective épidémiologique qui considère ces jeunes comme un groupe à risque qu'il faut éduquer, réhabiliter ou protéger. Ce type de recherche, surtout présent en Amérique du Nord ${ }^{2}$ a pour objectif de connaître les comportements des jeunes de la rue, afin d'y apporter une intervention spécifique, notamment en termes de prévention (Unger et al., 1998; Agence de santé publique du Canada, 2006a; Selsnick et al., 2006). L'accent y est mis sur des données de type quantitatif et elles ont l'avantage de mettre en évidence un certain nombre de tendances et de comportements caractéristiques des jeunes de la rue. Ce type de recherche ne manque pas d'influencer le discours politique dans ce domaine.

Parallèlement à cette association des jeunes à des comportements qui présentent un risque pour eux-mêmes, on voit apparâtre une interprétation du risque que leurs comportements engendrent pour le reste de la société, incluant l'apparition de la notion d'incivilité (Coutant, 2005). Cette interprétation du risque fait appel à une gestion sécuritaire de cette population, visant davantage à faire respecter l'ordre public qu'à s'intéresser aux problèmes vécus par ces jeunes. Or, comme le rappelle Stoecklin $(2000: 41)$ : «La définition d'une "population à risque" est le résultat provisoire des luttes d'influence entre les institutions sociales qui revendiquent la capacité de s'en occuper.» Ce, dans un contexte sociopolitique qui privilégie depuis une trentaine d'années une logique

2. Si elle concerne surtout les recherches en Amérique du Nord, depuis quelques années, l'approche épidémiologique se retrouve aussi dans les recherches concernant les enfants de la rue des villes d'Afrique, d'Amérique latine, d'Asie et d'Europe de l'Est (Aral et al., 2005; Oyefara, 2005; Dickson-Gomez et al., 2006). Si elle y est moins présente, cette tendance n'épargne pas l'Europe. Damon (2002) montre par exemple comment l'intervention auprès des sans-abri, considérés comme les "exclus des exclus», a opéré un glissement de la répression à l'éducation, sans pour autant que la dimension sécuritaire ne soit totalement évacuée. 
de ciblage croissant des populations devant faire l'objet d'interventions ou de protection spécifiques, dans une optique d'efficacité et de contrôle des coûts notamment (Damon, 2002: 16-17; Borgetto, 2003).

On constate par ailleurs que dans cette perspective, les notions de choix et d'intentions propres aux jeunes n'interviennent pas ou peu. Bessant (2001: 41) suggère que le recours au comportement à risque pour caractériser les jeunes de la rue viendrait se substituer au recours aux notions de délinquance et d'inadaptation sociale utilisées par un certain courant de la sociologie de la déviance, associé notamment à l'École de Chicago (Trasher, 1927). En ce sens, l'approche épidémiologique, tout comme l'approche sécuritaire, perpétuerait une vision des jeunes de la rue considérés incapables de donner eux-mêmes un sens à leur vie de rue, seul le chercheur étant compétent pour la définir en termes de risque ou de danger. Même si certains chercheurs introduisent un volet psychosocial à leurs travaux (Roy et al., 2005), ce sont surtout les comportements qui comptent, compris comme des unités d'observation que l'on décrit dans une perspective sociosanitaire. Bien que ces recherches nous informent des problèmes vécus par les jeunes de la rue et que ces données puissent être essentielles pour les services d'intervention spécialisés dans les différentes problématiques mentionnées (toxicomanie, santé mentale, HIV, etc.), elles permettent difficilement de saisir les enjeux subjectifs associés à la vie de rue. En effet, comme le souligne Parazelli (2000: 2):

Bien que nécessaires à la connaissance du phénomène, les caractéristiques même détaillées d'un groupe social ainsi que l'inventaire global des problèmes vécus par les jeunes de la rue ne nous informent pas d'emblée sur les enjeux de son existence sociale ni sur la dynamique interne de ses membres.

L'importance de ce type d'approche est révélatrice de celle de la notion de risque dans les représentations à l'égard des jeunes de la rue, ce qui conduit à justifier l'adoption de politiques de plus en plus axées sur la sécurité, à la fois sur les plans de la santé publique et de l'ordre public, dans le but de prévenir à tout prix les risques que ces jeunes encourent ou qu'ils font encourir au reste de la société. Au point où l'on peut se demander avec Coutant (2005) si ces jeunes ne sont pas victimes de «délit de jeunesse».

Si le risque fait peur au point de vouloir absolument le prévenir ou le gérer, il constitue par ailleurs une valeur centrale de l'idéologie 
individualiste qui caractérise nos sociétés occidentales actuelles. Associé à la créativité et à l'initiative, il est ainsi en même temps valorisé et même encouragé dans une perspective de performance, de différenciation et d'expérimentation de soi et de ses limites. Cette représentation $\mathrm{du}$ risque prend particulièrement du sens à l'adolescence, dont on peut dire qu'elle constitue un risque en soi. Un «risque nécessaire» même, puisque dans le contexte de transition, d'expérimentation et de découverte qui caractérise cette période, la prise de risque permet la connaissance de soi et l'expérience de ses limites (Le Breton, 2002; Jeffrey et al., 2005).

Face à l'abondance de données épidémiologiques concernant les risques que les jeunes encourent ainsi qu'aux statistiques qui annoncent l'augmentation inexorable de la délinquance juvénile, on peut avoir tendance à oublier que ces comportements, même s'ils représentent souvent un risque bien réel qu'il ne s'agit pas de nier, constituent en même temps les manifestations d'enjeux plus profonds, en lien avec les défis propres à la jeunesse contemporaine. À partir des propos de jeunes sortis de la rue interrogés à Montréal ${ }^{3}$, cet article vise à mettre en lumière l'écart entre le sens attribué par les jeunes à la marginalité et à la normalité et le discours épidémiologique et sécuritaire que l'on retrouve dans une partie importante de la recherche scientifique et sur le plan politique, ainsi que les effets de ce dernier en termes de reconnaissance sur leur processus de sortie de la rue. Il ne s'agit pas ici de nier les risques réels encourus, mais au contraire de proposer des points de repère pour l'intervention au plus proche de la réalité vécue par ces jeunes. C'est dans ce sens que sont proposées des pistes de lectures alternatives de leurs comportements, à travers quelques exemples analysés à l'aide d'une approche interactionniste (Mead, 1934), complétée par des apports de la psychanalyse (Winnicott, 2004), permettant de mettre en lumière la quête identitaire à l'œuvre dans l'appropriation et la sortie de la rue et les dynamiques de reconnaissance qui y sont associées.

3. Cette enquête a été réalisée dans le cadre d'une thèse de doctorat portant sur le rôle de la reconnaissance dans le processus de sortie de la rue des jeunes à Montréal (Colombo, 2008). Privilégiant le point de vue des jeunes qui sont sortis de la rue, 24 entrevues individuelles ont été menées. L'objectif était d'identifier les conditions de reconnaissance qui permettent le repositionnement identitaire amenant le sujet-acteur à sortir de la rue. Ces entrevues ont fait l'objet d'une analyse de contenu approfondie à partir d'une grille dans le but de mettre à jour les dynamiques identitaires associées aux trois formes de reconnaissance (affective, sociale et juridique) identifiées par Honneth (2002). Ces résultats ont été soumis pour validation aux répondants lors de deux focus groupes. 


\section{Jeunesse et risque}

L'apparition d'appellations telles que "groupe à risque» ou «jeunes vulnérables» traduit une multiplication bien réelle de comportements de plus en plus risqués de la part des jeunes. Le problème n'est pas tant de mettre à jour ces nouvelles formes de souffrance juvéniles que de réduire leurs pratiques à leur seul aspect de dangerosité. Nous proposons de comprendre ces dernières à la lumière d'enjeux particuliers qui caractérisent le passage à l'âge adulte dans nos sociétés occidentales contemporaines. En effet, les sociologues de la jeunesse (Galland, 1993 ; Gauthier, 2000; Bajoit, 2005) s'accordent pour dire que l'incertitude associée à cette période de la vie est aujourd'hui exacerbée par la multiplication des repères normatifs qui caractérisent nos sociétés dites individualistes. On assiste à un éclatement des repères permettant au jeune de se reconnaitre et d'être reconnu dans une position d'adulte autonome. Dans ce sens, Dubar (2000) soutient qu'on ne peut plus parler de socialisation comme d'un «modelage» passif des jeunes par les institutions ou d'une action unilatérale des adultes sur les jeunes. Dans le contexte social actuel, la socialisation correspondrait plutôt à une individuation marquée par le souci de trouver sa voie à travers l'expérimentation. La prise de risque est alors considérée comme un moyen d'explorer ses propres potentialités en s'engageant dans des défis dont la nature et le degré de dangerosité varient selon les jeunes et dont l'issue peut même être mortelle ou laisser des séquelles importantes sur le plan de la santé mentale ou physique.

Sans nier les dangers associés à ces pratiques, les travaux anthropologiques de Le Breton (2002), ainsi que d'autres à sa suite (Jeffrey et al., 2005), permettent de comprendre dans les conduites à risque adoptées par certains adolescents des pratiques qui s'apparentent à des rites de passage personnalisés. Ne trouvant pas, dans les institutions traditionnelles de socialisation, des repères qui fassent suffisamment de sens à leurs yeux pour s'approprier une place sociale, certains jeunes se soumettraient, de leur propre initiative, à des épreuves qui leur permettraient de se «prouver» qu'ils sont de «vrais adultes».

C'est dans ce sens que l'on peut comprendre plusieurs pratiques associées à l'appropriation de la rue par certains jeunes. Âgés de 14 à 25 ans environ, ces jeunes s'approprient certains lieux urbains et y développent des pratiques culturelles et sociales variées, parmi lesquelles on trouve notamment des pratiques dites à risque. Il peut s'agir des risques que les jeunes encourent eux-mêmes, comme dans le cas des 
pratiques de toxicomanie, de prostitution, de marquage du corps ou d'automutilation, de comportements suicidaires ou de sexualité non protégée. Il peut aussi s'agir de risques qu'ils font encourir à d'autres, comme dans le cas des «incivilités» (allant des tags à la mendicité, en passant par le squeegee $e^{4}$, de la vente de drogues, de comportements agressifs ou de vols, voire de l'insertion dans des réseaux associés à des activités criminelles plus importantes.

En même temps qu'elles comportent une part de danger indéniable, nous allons voir que ces pratiques peuvent comporter une dimension constructive pour ces jeunes si on les associe à leurs efforts de socialisation, même si ceux-ci revêtent parfois des formes difficiles à décoder. En raison de contextes familiaux marqués par des relations de rejet, d'abandon ou d'incohérence, les jeunes interviewés dans le cadre de l'enquête n'ont pas pu trouver au sein de leur famille des repères suffisamment clairs pour leur permettre d'attribuer un sens aux normes sociales et d'organiser leur rapport au monde. Ne se sentant que partiellement ou pas reconnus au sein de leur famille et/ou des institutions traditionnelles de socialisation, la rue leur a semblé un lieu où ils pourraient échapper à ces dynamiques familiales insatisfaisantes. En même temps, elle comporte un potentiel d'indétermination qui leur permet d'être reconnus dans le rapport au monde paradoxal qu'ils ont développé. Ils tentent à la fois d'échapper à des dynamiques relationnelles destructrices, mais ne peuvent s'empêcher de les reproduire dans un mouvement de repli sécurisant.

Nous avons montré ailleurs (Colombo, 2008) comment un tel héritage familial, même précaire, influence de façon importante les trajectoires de ces jeunes en les amenant à développer des attentes de reconnaissance spécifiques associées à des modes particuliers de relation à la rue, puis à la sortie de la rue. Sans entrer dans le détail de ces différentes dynamiques relationnelles, nous montrerons ici, à partir de la lecture qu'ils en font eux-mêmes une fois sortis de la rue, que les pratiques dites à risque qu'ils ont adoptées lorsqu'ils étaient dans la rue s'inscrivent dans une perspective de construction identitaire associée à leur quête de reconnaissance. Le rapport à de telles pratiques est toujours paradoxal et l'on ne peut vraiment saisir le sens qu'elles revêtent à leurs yeux que si on les considère à la fois dans leur dimension constructive (quête identitaire) et destructive (risques réels et souffrances).

4. Activité consistant à laver les pare-brises des voitures en échange d'argent. 


\section{Les figures du risque dans la rue}

Comme l'exprime une répondante, le risque associé à la rue comporte une dimension à la fois effrayante et attirante, parce qu'il est source d'incertitude, mais aussi de liberté associée à une certaine imprévisibilité:

Mais c'est la peur du non-connu... on dirait que la peur, y a comme une attirance envers ça. Qu'est-ce qui n'est pas à tous les jours. Ce qui est attirant, c'est que tu sais pas ce qui va t'arriver le lendemain. Tandis que rester chez toi, tu vas à l'école à tous les jours, tu sais qu'est-ce qui va arriver. (Camille ${ }^{5}$, dans la rue de 16 à 20 ans)

Ce qui est considéré comme attirant ici est le caractère imprévisible des actions qui pouvaient survenir dans la rue, susceptibles de redonner un certain sens à une existence dont le quotidien est devenu trop prévisible, au point d'être banal et ennuyeux. Cette imprévisibilité fait référence à ce que Winnicott (2004) nomme «l'indétermination des règles du jeu», nécessaire à tout espace d'expérimentation sociale permettant une appropriation de leurs actes par les jeunes. Contrairement à l'imprévisibilité d'actes parentaux incohérents, dont plusieurs jeunes se sentaient captifs, le caractère imprévisible de la rue est davantage lié à un imaginaire d'aventure censé leur offrir toute la liberté d'accomplir leurs expérimentations de façon autonome.

Les adultes ne semblant pas en mesure d'assumer leur rôle de transmission sociale, ces jeunes sont amenés à trouver par eux-mêmes les moyens de compléter leur socialisation, selon ce que Parazelli (2002: 272) nomme un «imaginaire d'autonomie naturelle». Selon cet imaginaire, l'autonomie pourrait s'acquérir sans l'aide des adultes, de façon presque naturelle, à travers l'expérimentation individuelle ou au sein du groupe de pairs. C'est pourquoi le potentiel d'exploration en marge de toute institution traditionnelle de socialisation que semble contenir la rue leur paraît attirant, car il leur permet d'expérimenter «en vrai»ce que d'autres apprennent sur les bancs d'école. Les mots d'une autre répondante font penser à l'expression «les voyages forment la jeunesse», dans laquelle les voyages seraient remplacés, dans son expérience, par la consommation de drogues ${ }^{6}$ :

5. Afin de préserver l'anonymat des personnes interviewées, les prénoms utilisés sont fictifs.

6. Il va sans dire que les deux expériences ne sont par ailleurs pas comparables sur le plan des dangers et des souffrances vécues. 
Tsé, il y en a qui tripent: «Je vais aller faire le tour du monde», mais moi j'ai tripé: «Je vais essayer de me piquer un peu.» C'est niaiseux hein, c'est ça! Je voulais voir c'était quoi. Parce que j'ai vu plein de films là-dessus, j'avais joué une pièce de théâtre [...] pis ça m'intriguait. Je suis allée le vivre, je suis allée le voir. (Stéphanie, dans la rue de 18 à 20 ans)

La consommation de psychotropes constitue une pratique particulièrement répandue chez les jeunes de la rue. Il s'agit toutefois d'être prudent et de ne pas généraliser cette association entre rue et consommation de drogues, puisque certains jeunes de la rue en consomment très peu ou se limitent aux drogues douces. Par ailleurs, les interactions entre ces deux dynamiques varient selon les trajectoires (parfois, la toxicomanie précède la vie de rue, d'autres fois, c'est l'inverse; parfois l'expérience de toxicomanie est parallèle à la vie de rue, d'autres fois, elle y est intimement liée). En outre, comme l'a également analysé Moïse (2000), le choix des produits consommés et des modes de consommation n'est pas le fruit du hasard, mais il peut être mis en relation avec le sens que les jeunes donnent à leur vie de rue. La consommation exploratoire et curieuse des débuts dans la rue, poussant à expérimenter divers produits, peut souvent céder le pas à une consommation plus destructrice et marquée par une prise de risque ainsi qu'une dépendance plus importante.

En ce qui concerne spécifiquement la consommation de drogues par injection, nous avons pu constater, à l'instar de Parazelli (1997: 416), que le sentiment de manque relationnel que peuvent vivre les jeunes de la rue, suite à des relations parentales difficiles, suscite chez plusieurs d'entre eux le désir de dépendance à un objet, qui peut aller jusqu'à la fusion rassurante. On existe à travers la reconnaissance des autres. Ainsi, lorsque la relation avec l'autre fait défaut, comme c'est le cas lorsque les parents sont absents, la possibilité d'être reconnu en tant que sujet peut poser problème. C'est pourquoi certains jeunes peuvent être amenés à créer l'illusion d'une relation à l'autre par l'intériorisation d'une substance. Provenant du monde extérieur, la substance psychotrope fait alors office d'«autre d $^{7}$, comme l'exprime cette répondante à travers la métaphore du mariage:

Comme exemple la fois que je me suis shootée, j'avais rencontré quelqu'un [...], pis je l'avais vu se shooter, pis c'était comme féerique un peu. Quand son sang s'est mélangé avec la substance, il disait: «Mariage.» Fait que ça

7. Il s'agit là toutefois d'une illusion puisque la substance n'a qu'une valeur utilitaire qui ne peut être source de reconnaissance (Jeammet, 1993). 
m'est resté dans la tête un peu. Jusqu'à ce que je me suis dite: «Bon ben je vais essayer.» (Camille, dans la rue de 16 à 20 ans)

Dans certains cas, la consommation de psychotropes, comme d'autres pratiques qui mettent à mal le corps (malnutrition, automutilation, etc.), s'inscrit davantage dans une dynamique de renchérissement de la souffrance, voire de poursuite de la mort, comme s'il s'agissait de matérialiser la douleur et le sentiment de non-existence vécu dans la relation aux autres et plus particulièrement aux parents (Gilbert, 2004). L'idée est alors de s'anesthésier, de ne plus se sentir: "[Je consommais] de la coke, l'héroïne, tout, PCP, tout, tout! N'importe quoi, en autant que je me sente pas» (Frédéric, dans la rue de 14 à 24 ans). C'est comme si, en maltraitant leur propre corps, ces jeunes s'appropriaient le rejet et la destruction de soi dont ils ont fait l'objet durant leur enfance, renchérissant même sur ces dynamiques. Sans parler des appels à la reconnaissance que peuvent constituer, de façon détournée et parfois inconsciente, ces pratiques de destruction de soi (Poirier et al., 1999). Dans d'autres cas, de telles pratiques s'inscrivent moins dans une logique de renchérissement de la destruction de soi amorcée dans la relation parentale que dans une logique de test de ses propres limites. En effet, selon Le Breton (1991: 17), la limite est une nécessité anthropologique qui permet d'exister en se situant par rapport à des repères symboliques délimités. C'est comme si, à défaut de repères symboliques clairs pour délimiter leur identité, certains jeunes avaient besoin de mettre à jour leurs limites physiques, en maltraitant leur corps, en le poussant à bout. Comme cette jeune qui se sentait «complètement déchirée en dedans» et est partie nu-pieds dans la neige avec un inconnu: «[...] on a baisé pis on a fait une grosse ligne de PCP. J'ai pensé, ça, ça va m'aider» (Valentine, dans la rue de 18 à 19 ans). Ou encore cette autre répondante qui explique qu'à la fin de son parcours de toxicomanie, ce n'était plus vraiment les sensations liées à la substance qu'elle recherchait, mais le geste de s'injecter qui était devenu pour elle un rituel dont elle n'arrivait pas à se défaire, dans une logique se rapprochant de l'automutilation ${ }^{8}$ où la sensation de l'aiguille sur la peau importe davantage que l'injection. Comme si elle avait besoin de mettre à l'épreuve les limites de son corps, de sentir la résistance de la peau à travers la douleur pour attester qu'elle était toujours là, de vérifier la limite entre son monde intérieur et le monde extérieur pour bien les distinguer (Aubin, 2000). Pour

8. Nous reprenons là ses propres termes. 
d'autres, ce jeu avec la mort est perçu comme un défi, dans lequel il s'agit de faire l'expérience de ses limites dans une compétition avec soi-même. En se mettant à l'épreuve à travers la vie de rue vécue comme un défi, ces jeunes se créeraient leurs propres critères de légitimation de leur existence, puisqu'ils ont hérité de repères trop incohérents pour donner du sens à cette dernière. Cette mise à l'épreuve de soi a pris des formes diverses selon leurs trajectoires, comme nous avons pu le voir. Selon Le Breton (1991), dans bien des cas, la prise de risque vise à «charmer symboliquement la mort», afin de fixer les limites de la puissance de celle-ci, à l'image du rite moyenâgeux de l'ordalie. Le fait de poser un défi à la mort renforce la valeur de son existence, puisque la vie ressort gagnante de l'affrontement avec la mort.

Ces exemples indiquent que, si les pratiques à risque adoptées par les jeunes de la rue comportent un aspect destructif indéniable, cela n'exclut pas qu'elles puissent également révéler des dynamiques qui relèvent d'un effort de construction identitaire. Si les adultes se doivent de reconnaître la souffrance et les dangers encourus par ces jeunes, il est toutefois important qu'ils ne se limitent pas à une attitude axée sur la sécurité et qu'ils reconnaissent également la quête identitaire que révèlent ces comportements. En effet, comme le souligne Jeffrey (2005: 48), même si elles sont bricolées à l'écart des institutions traditionnelles de socialisation, ces épreuves que s'imposent ces jeunes ne peuvent jouer leur rôle de rituel de passage que s'ils sont reconnus par les autres et particulièrement par les adultes:

Lâge adulte se mérite, mais il doit être reconnu. Lorsque le rite n'est plus sous la gouverne des aînés et que chaque jeune est laissé à lui-même pour accéder au statut d'adulte, le passage devient périlleux et risque de se prolonger.

Toutefois, l'image du risque véhiculée par les adultes à travers une interprétation de ces pratiques en termes de risques pour la santé ou d'incivilités comporte le danger d'occulter toute la part de bricolage identitaire qui peut s'y jouer. À ce propos, Tursz (1993: 147) se demande si les adultes n'ont pas tendance à projeter sur les jeunes des peurs qui sont plutôt les leurs. Elle constate en effet que les adultes ont souvent du mal à accepter la jeunesse et le pouvoir de prendre des risques qu'ils ont eux-mêmes perdus et à renvoyer par conséquent aux jeunes une image négative du risque. Il ne s'agit pas ici d'encourager une attitude de laisser-faire de la part des adultes, mais bien au contraire 
de plaider pour une meilleure prise en compte du sens que les pratiques à risque peuvent revêtir aux yeux des jeunes, afin d'éviter une radicalisation de celles-ci.

\section{La sortie de la rue est-elle une sortie du risque?}

L'écart entre les interprétations adultes des pratiques à risque et le sens qu'elles peuvent prendre pour les jeunes n'est pas sans incidence sur la sortie de la rue. Les résultats de la recherche que nous avons menée sur les sorties de la rue montrent que l'attractivité des lieux urbains et des pratiques associées à la vie de rue diminue après un certain nombre d'années et que d'autres lieux et d'autres pratiques deviennent plus attractifs pour ces jeunes ${ }^{9}$. Ce changement d'investissement témoigne d'un repositionnement identitaire, qui est fortement associé aux manifestations de reconnaissance (Honneth, 2000) que le jeune a pu s'approprier pour construire sa nouvelle position identitaire (Colombo, 2008). Pour ces jeunes, il s'agissait à la fois d'être reconnus en tant que jeunes de la rue et comme étant capables d'occuper une autre position identitaire. Dans ce sens, le processus de sortie de la rue a pu s'amorcer lorsque le jeune a pu établir des relations dans lesquelles il a senti qu'on reconnaissait le sens des pratiques à risque qu'il adoptait, tout en parvenant à être reconnu différemment, en établissant un rapport à luimême et aux autres qui soit plus satisfaisant. Par exemple, pour certains jeunes, la sortie de la rue ne pouvait s'amorcer qu'après avoir atteint leur limite, que ce soit en frôlant la mort ou en se trouvant confrontés à une image d'eux-mêmes qui les conduit brutalement, d'une part, à perdre le sentiment d'omnipotence qui peut caractériser l'adolescence, et d'autre part, à réaliser que la vie de rue et les pratiques associées ne correspondaient plus à leurs attentes de reconnaissance:

Le docteur se posait des questions, comment ça se faisait que j'avais toughé longtemps de même. Tsé, j'ai frôlé la mort, je suis allé en prison, quelque part, je veux pas mourir. À quelque part, j'aimais pas la vie, mais là je l'aime

9. Soulignons que l'échantillon de l'étude est limité à des jeunes dont la sortie de la rue a été un succès. Ceci constitue une limite de l'étude mais également l'un de ses aspects originaux, la plupart des études portant sur des jeunes encore dans la rue, à peine sortis ou pris en charge par des institutions (Colombo, 2008). Plusieurs recherches indiquent néanmoins que la plupart des jeunes de la rue s'en sortent (Agence de la santé publique du Canada, 2006b; Levac, 2006). Seule une minorité d'entre eux y laissent leur peau ou intègrent des réseaux plus criminalisés, et très rares sont ceux qui rejoignent les rangs des itinérants plus âgés. 
en hostie! Quand tu passes comme ça proche de la mort, t'apprécies la vie en sale! Quand tu te réveilles pis, hostie, je suis pas mort! Wow! Allume le cave, change-là ta vie! (Vincent, 25 ans, sorti depuis 2 ans)

Dans d'autres cas, la demande de reconnaissance porte davantage sur leur attachement à la rue et à un mode de vie plus marginal, qu'ils ne considèrent pas comme incompatible avec leur sortie de la rue. En effet, pour certains jeunes qui ont plutôt tendance à se méfier des relations avec des adultes (de peur, par exemple, de vivre d'autres abandons), il est néanmoins très important que soit au moins reconnu leur attachement à leurs pairs. Comme dans le cas de cette jeune qui a été éloignée contre son gré de son groupe de pairs et qui a éprouvé le besoin de retourner auprès d'eux vérifier la solidité de leurs liens: «Fait que pour moi c'était un passage obligé de vérifier que c'était pas du vent. Mon identité pendant dix ans, viens pas me dire que c'est du vent!» (MarieJo, 28 ans, sortie depuis 12 ans). Leur sortie de la rue consiste par conséquent davantage en une redéfinition de leurs relations avec leurs pairs ainsi qu'avec la rue qu'à une coupure radicale avec ce milieu. Tout en composant avec le risque de rechute que comporte une telle proximité avec la rue.

Parfois, la sortie de la rue passe par des voies ambiguës, voire risquées pour le jeune. Comme dans le cas de ces jeunes hommes qui s'en sont sortis grâce à leur relation avec un sugar daddy. À première vue, une telle relation avec un homme, souvent beaucoup plus âgé qu'eux et qui les entretient en échange de leurs services sexuels, peut apparaître davantage destructive que constructive. En effet, la plupart de ces jeunes hommes n'étant pas homosexuels, ils reproduisent dans ces relations une forme de négation d'eux-mêmes. D'ailleurs, l'incohérence de telles relations débouche le plus souvent soit sur une rupture, soit sur une redéfinition de la relation, qui peut par exemple perdre tout caractère sexuel avec le temps. Mais en même temps, ces relations peuvent faciliter la sortie de la rue de ces jeunes. Elles sont significatives à leurs yeux, du moins durant un certain laps de temps, car ils y trouvent une réponse à leurs attentes de reconnaissance, que ce soit de leurs qualités esthétiques et sexuelles, de valorisation à travers un train de vie luxueux, du sentiment d'exister en tant qu'objet d'amour ou encore d'investissement d'une relation qui peut comporter les traits d'une relation parentale et qui leur apparaît davantage satisfaisante que celle avec leurs parents (Moïse, 2002; Dorais, 2003). Cette ambiguiité ressort bien des propos de ce répondant: 
I: Mais, comment tu qualifierais votre relation à ce moment-là ?

$\mathrm{R}$ : Ben c'est un sugar daddy ben raide là! «Tu me donnes de l'argent...» Mais tsé, il m'écoutait, on parlait beaucoup, on allait au cinéma, on allait manger ensemble. Tsé, pour moi, c'était comme mon père. C'était pas sain pantoute. C'était une relation malsaine. Parce que lui, il s'attendait à des faveurs sexuelles, pis moi je m'attendais à des faveurs paternelles. Fait que les deux, on s'attendait à deux choses totalement différentes. C'est pour ça que ça a pas fonctionné. Mais ça a quand même porté fruit à quelque part, parce qu'il m'a sorti de la rue le bonhomme. C'est lui, il m'a bâti un pont solide. Il m'a dit: «Tiens, regarde, t'as plus à vivre dans la rue.» (Vincent, 25 ans, sorti depuis 2 ans)

Notre propos ici ne consiste pas à valoriser de telles pratiques, ni à encourager une attitude de laisser-faire, mais plutôt à offrir des repères pour l'intervention à partir d'une lecture paradoxale de ces pratiques (à la fois destructrices et constructrices). Ces exemples montrent que dans certains cas, la volonté d'éviter à tout prix les prises de risque de ces jeunes comporte le danger de voir leur quête de limites se prolonger, voire se dérouler dans des lieux encore plus à l'écart du monde des adultes.

\section{Conclusion}

Avec la modernisation et la laïcisation de la société, plusieurs rituels, dont ceux associés au passage à l'âge adulte, ont disparu ou se sont dilués, laissant les jeunes relativement livrés à eux-mêmes dans cette transition. Pour des jeunes ayant vécu des relations parentales marquées par l'incohérence, le rejet ou encore l'abandon, la difficulté de trouver $\mathrm{du}$ sens dans les repères proposés par les institutions traditionnelles de socialisation ou par les médias (y compris l'internet, la publicité, etc.) est encore plus importante. Dans ce contexte, certains jeunes s'imposent des épreuves parfois très risquées, en adoptant par exemple un mode de vie associé à la rue. Il s'agit de reconnaître la part de danger importante de ces épreuves, qui peuvent se solder par la mort ou laisser des séquelles sur le plan de leur santé physique ou psychique. Mais il s'agit également de les comprendre comme une tentative de créer à leur façon les balises de ce passage à l'âge adulte, à travers l'exploration, la recherche de limites ou encore la mise en scène de conditions familiales que l'on cherche à se réapproprier. Il existe plusieurs exemples d'intervention à Montréal qui prennent le risque de reconnaître cette part de quête 
à travers de telles pratiques, sans pour autant nier la part de danger réel qu'elles comportent. Mentionnons notamment le travail de rue, les approches de réduction des méfaits, le Collectif d'intervention par les pairs, où l'intervention se fait par d'anciens jeunes de la rue, ou encore le Dispositif Mendel, où policiers, intervenants, élus et jeunes de la rue communiquent de façon démocratique.

On observe toutefois une tendance dans la sphère politique à privilégier une lecture des pratiques de ces jeunes en termes de nuisances, aussi bien sur le plan sociosanitaire que sur le plan de l'ordre public. Dans ce contexte, on peut se demander lequel des comportements est le plus risqué: ces pratiques juvéniles ou la tendance à l'obsession sécuritaire? Celle-ci comporte le risque de nier toute la part de bricolage que cachent les pratiques de ces jeunes et qui leur permet de construire, tant bien que mal, leur processus de sortie de la rue.

\section{Références}

Agence de santé publique du Canada (2006a). Infections transmises sexuellement chez les jeunes de la rue au Canada. Constatations découlant de la surveillance accrue des jeunes de la rue au Canada, 1999-2003. Ottawa: Agence de santé publique du Canada.

Agence de santé publique du Canada (2006b). Les jeunes de la rue au Canada. Constatations découlant de la surveillance accrue des jeunes de la rue au Canada, 1999-2003. Ottawa: Agence de santé publique du Canada.

Aubin, D. (2000). Le corps, lieu de repères pour les jeunes de la rue ou la quête d'un territoire d'appartenance. Santé mentale au Québec, $X X V(2), 90-108$.

Bajoit, G. (2005). Les jeunes en quête de sens dans un monde incertain. Conseil de développement de la recherche sur la famille du Québec. Hiver 2005, numéro spécial, 2-3.

Borgetto, M. (2003). Identification, problèmes et enjeux du ciblage. Informations sociales, 108, 4-7.

Colombo, A. (2008). La reconnaissance: un enjeu pour la sortie de la rue des jeunes à Montréal. Thèse de doctorat en Études urbaines. Montréal: Université du Québec à Montréal.

Coutant, I. (2005). Délit de jeunesse. La justice face aux quartiers. Paris: La Découverte.

Damon, J. (2002). La question SDF: critique d'une action publique. Paris: PUF.

Dorais, M., \& Lajeunesse, S. L. (2003). Intimité à vendre? Comment devient-on travailleur du sexe? Sociologie et sociétés, 35 (2), 121-138.

Galland, O. (1993). Adolescence et post-adolescence: la prolongation de la jeunesse. In A. Tursz, Y. Souteyrand \& R. Salmi (eds.), Adolescence et risque (29-40). Paris: Syros. 
Gauthier, M. (2000). L'âge des jeunes: «un fait social instable». Lien social et politique - RIAC, 43, 23-32.

Gilbert, S. (2004). L'idéal du moi comme point de mire et le social en toile de fond: Une comprébension de la dynamique sociopsychique de l'itinérance des jeunes adultes. Thèse de doctorat en psychologie, Université du Québec à Montréal.

Honneth, A. (2000). La lutte pour la reconnaissance. Paris: Les éditions du Cerf.

Jeammet, P. (1993). L'adolescence est-elle un risque? In A. Tursz, Y. Souteyrand $\&$ R. Salmi (eds.), Adolescence et risque (41-51). Paris: Syros.

Jeffrey, D. (2005). Conduites à risque et rites de passage à l'adolescence. In D. Jeffrey, D. Le Breton \& J.-J. Lévy (eds.), Jeunesse à risque. Rite et passage (45-56). Ste-Foy: PUL.

Le Breton, D. (1991). Du risque à l'ordalie. In D. Le Breton (ed.), Passions du risque (16-47). Paris: Métaillé.

Le Breton, D. (ed.) (2002). L'adolescence à risque. Paris: Hachette littératures.

Levac, Ch. 2006. La rue, un chemin tracé d'avance? Montréal: Le Refuge des jeunes de Montréal.

Mead, G. H. (1934) [édition de 1963]. L'Esprit, le Soi et la Société. Paris: PUF.

Moïse, J. (2000). Psychotropes et jeunes de la rue. Rapport. Montréal: L'intervenant.

Moïse, J. (2002). Adolescence, initiation et prostitution. Montréal: Éditions du Mistral.

Parazelli, M. (2000). L'encombrement socio-symbolique des jeunes de la rue au centre-ville de Montréal. Le cas d'un quartier en revitalisation: le Faubourg Saint-Laurent. In J.-P. Augustin \& C. Sorbets (eds.), Lieux communs. Aperçus sur l'aménagement de places et de parcs au Québec (169-199). Talence (France): Maison des sciences de l'Homme d'Aquitaine.

Parazelli, M. (2002). La rue attractive. Parcours et pratiques identitaires des jeunes de la rue. Sainte-Foy: Presses de l'Université du Québec.

Poirier, M., V. Lussier, Letendre, R., Michaud, P., Morval, M., Gilbert, S., \& Pelletier, A. (1999). Relations et représentations interpersonnelles de jeunes adultes itinérants. Au-delà de la contrainte de la rupture, la contrainte des liens. Rapport de recherche du GRIJA. Montréal: GRIJA.

Roy, É., Haley, N., Godin, G., \& Boivin, J.-F. (2005). L'bépatite C et les facteurs psychosociaux associés au passage à l'injection chez les jeunes de la rue. Rapport d'étape numéro 4. Montréal: Direction de la santé publique.

Selsnick, N., Bartle-Haring, S., Glebova, T., \& Glade, A. (2006). Homeless Adolescent Parents: HIV Risk, Family Structure and Individual Problem Behaviors. Journal of Adolescent Health, 39 (5), 774-777.

Stoecklin, D. (2000). Enfants des rues en Chine. Paris: Éditions Karthala.

Trasher, F. (1927). The Gang. Chicago: University of Chicago Press.

Tursz, A. (1993). Des diverses approches du risque à l'adolescence. In A. Tursz, Y. Souteyrand \& R. Salmi (eds.), Adolescence et risque (135-149). Paris: Syros. 
Unger, J. B., Simon, T. R., Newman, T. L., Montgomery, S. B., Kipke, M. D., \& Albornoz, M. (1998). Early adolescent street youth: An overlooked population with unique problems and service needs. Journal of early adolescence, $18(4), 325-348$.

Winnicott, D. W. (2004). Jeu et réalité. L'espace potentiel. Paris: Gallimard.

ABSTRACT - When we see the numerous risks that young people face on the street or cause for others, one can forget that the so called "at risk» behaviour are displays of deeper lying issues. While the ideal of individual auto-realization values certain forms of at risk behaviour, one can also discern the existence in our societies an obsession with safety that targets at risk populations. Based on a survey conducted in Montreal with street youth, this article aims to clarify the difference between the meaning attributed by youth to marginality and normality and the political tendency to favour epidemiological and safety oriented approaches toward these populations. It concludes with an analysis of the effects of these approaches in relation to the recognition on the youth street exit process.

KEYWORDS - At risk youth, street youth, epidemiology, safety, marginality.

RESUMEN - La abundancia de cifras sobre los riesgos que los jóvenes de la calle corren o hacen correr a otros puede hacernos olvidar que los susodichos comportamientos "de riesgo" no son más que manifestaciones de problemas más profundos. En efecto, al mismo tiempo que en que el ideal de realización individual se valorizan ciertas formas de toma de riesgo, en el seno de nuestras sociedades se presenta una obsesión sobre la seguridad centrada en la gestión de las poblaciones en riesgo. A partir de una investigación realizada con jóvenes que han dejado la calle en Montreal, este artículo busca ilustrar la divergencia existente entre el sentido atribuido por los jóvenes a la marginalidad y a la normalidad y las tendencias políticas a privilegiar el enfoque epidemiológico y de seguridad en relación con estas poblaciones, además del efecto que ello tiene sobre estas últimas en términos de reconocimiento del proceso de retiro de estos jóvenes de las calles.

PALABRAS CLAVE - Jóvenes en riesgo, jóvenes de la calle, epidemiología, seguridad, marginalidad. 